\title{
Weber's Law Modeled by the Mathematical Description of a Beam Balance
}

\author{
RICHARD G. LANZARA \\ Department of Allied Health Sciences, \\ The City University of New York and Bio Balance Inc., \\ New York, New York
}

Received 9 July 1993; revised 1 November 1993

\begin{abstract}
A beam balance is analyzed as a model that describes Weber's law. The mathematical derivations of the torques on a beam balance produce a description that is strictly compatible with that law. The natural relationship of the beam balance model to Weber's law provides for an intuitive understanding of the relationship of Weber's law to sensory and receptor systems. Additionally, this model may offer a simple way to compute perturbations that result from unequal effects on coupled steady-state systems. A practical outgrowth from this work is that a relatively simple mathematical description models sensory phenomena and may aid in the understanding of sensory and receptor systems.
\end{abstract}

\section{INTRODUCTION}

Weber's law and the modified Weber-Fechner law have been considered mathematical descriptions of sensory responses for more than 100 years. A large number of sensory systems and their respective stimuli, including sound, light, smell, and taste stimuli, can be modeled in the moderate range of stimuli by these laws. However, deviations from predicted values have been observed for small and large stimulus intensities [3]. The failure of Weber's law and the Weber-Fechner law to model the extremes of responses to either high or low stimuli has generated attempts to find physically more meaningful models $[1-3,5]$.

Originally the German anatomist and physiologist E. H. Weber studied the response of humans to physical stimuli. He found that a person is unable to discriminate between 20.5- and 20.0-g weights in each hand but can usually discriminate between 21 and $20 \mathrm{~g}$. For a weight of $20 \mathrm{~g}$, the required increase of the stimulus for the just noticeable difference is $1 \mathrm{~g}$. However, with an initial weight of $40 \mathrm{~g}$, the 
result is quite different. A person cannot reliably discriminate between $41 \mathrm{~g}$ and $40 \mathrm{~g}$ but requires an increase of stimulus of $2 \mathrm{~g}$ for the just noticeable difference. Similarly, Weber found that 63 grams can be discriminated from 60 grams, 84 grams from 80 grams, and 105 grams from 100 grams. The required increase of the stimulus for the just noticeable difference in all cases is $5 \%$ of the original weight [1].

From these obscrvations in 1834 Weber formulated a law that establishes that for the just noticeable difference a given intensity of stimulation is proportional to the original stimulus. Remarkably similar results were discovered for sound, light, smell, and taste stimuli. In general it was discovered that if $s$ is the magnitude of a stimulus and jnd is the just noticeable difference for discrimination, then the ratio

$$
r=\frac{\text { jnd }}{s}
$$

is constant. The noticeable differences in sensation occur only when the increases (or changes) in stimuli are a constant percentage of the stimulus itself. This is Weber's law.

Fechner showed that joint acceptance of Weber's law plus the assumption that all jnd's have equal subjective size leads to a simple logarithmic rule relating the level of sensation to the stimuli

$$
\text { jnd }=A \log (s)+B,
$$

where $A$ and $B$ are fitted constants [1].

An important application of the Weber-Fechner law is the doseresponse relationship in biological assays. When the dose of a chemical (drug or hormone) is administered, the response is not linearly related to the dose; rather it is the logarithm of the dose versus the response that most nearly describes the dose-response relationship. Weber's law is approximately correct for a wide variety of sensory dimensions, although substantial deviations from predicted values are observed for either large or small stimuli.

\section{DERIVATION AND RESULTS}

The derivation begins with the torques on a beam balance as diagramed in Figure 1. The weights $w_{1}$ and $w_{2}$ at distances $d 1$ and $d 2$ from the fulcrum produce equal torques such that the balance is in an initial state of horizontal equilibrium. Therefore,

$$
w_{1}(d 1)=w_{2}(d 2)
$$



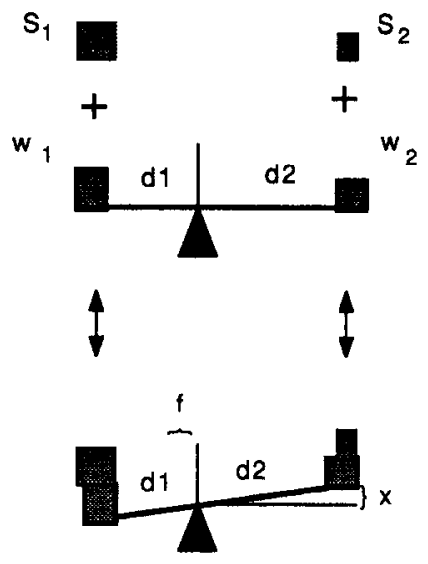

$$
w_{1}+s_{1} \quad w_{2}+s_{2}
$$
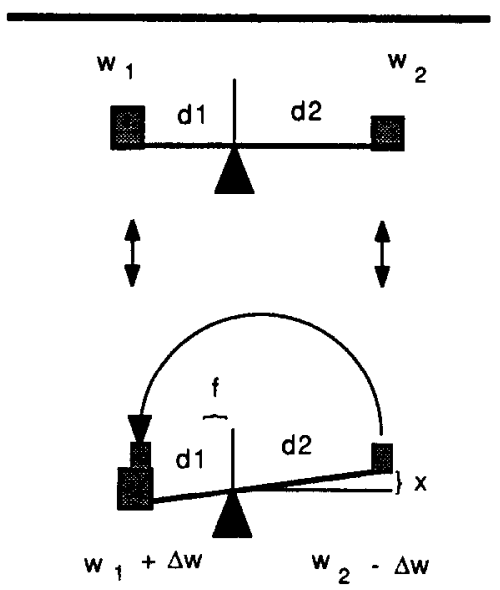

FIG. 1. A diagram illustrating two equivalent ways to produce equal displacements of a beam balance. (Top) The addition of weights $S_{1}$ and $S_{2}$ produces the displacement $x$. To establish horizontal equilibrium, the fulcrum is moved by the distance $f$ as pictured. (Bottom) The transfer of the weight $\Delta w$ from $w_{2}$ to the opposite side is diagramed. This produces an equal displacement $x$ as pictured and results in an equivalent movement of the fulcrum, $f$, to maintain the horizontal equilibrium.

If additional weights $S_{1}$ and $S_{2}$ are added to the opposite sides of the balance, a displacement $(x)$ will be produced if the torques are unequal. The fulcrum can be moved the distance $f$ as shown in Figure 1 to establish a new equilibrium point. The new equilibrium condition can 
be expressed as

$$
\left(w_{1}+S_{1}\right)(d 1-f)=\left(w_{2}+S_{2}\right)(d 2+f)
$$

or

$$
\frac{w_{1}+S_{1}}{w_{2}+S_{2}}=\frac{d 2+f}{d 1-f}
$$

An equivalent displacement can be produced by a transfer of some fraction of weight from one side of the balance to the other side. This is diagramed in Figure 1 and can be represented as

$$
\left(w_{1}+\Delta w\right)(d 1-f)=\left(w_{2}-\Delta w\right)(d 2+f)
$$

or by rearranging to give

$$
\frac{w_{1}+\Delta w}{w_{2}-\Delta w}=\frac{d 2+f}{d 1-f}
$$

where $\Delta w$ is the weight that is transferred from one side to the opposite side to produce a displacement that is equivalent to the previous displacement of the balance produced by the addition of weights $S_{1}$ and $S_{2}$. The right-hand sides of Equations (1) and (2) are identical; therefore,

$$
\frac{w_{1}+\Delta w}{w_{2}-\Delta w}=\frac{w_{1}+S_{1}}{w_{2}+S_{2}}
$$

Equation (3) shows that for equal displacements these ratios are equal for the two cases. That is, whether a fraction of the weight is transferred from one side to the opposite side or unequal torques are produced by the relative weighting produced by the simple addition of weights, the displacements of the balance from horizontal equilibrium can be made equal (see Figure 1). This relationship allows for a discrete and unique solution for $\Delta w$. Solving equation (3) for $\Delta w$ gives

$$
\Delta w=\frac{\left(w_{1}+S_{1}\right) w_{2}-\left(w_{2}+S_{2}\right) w_{1}}{\left(w_{1}+S_{1}\right)+\left(w_{2}+S_{2}\right)}
$$

or, upon simplification,

$$
\Delta w=\frac{S_{1} w_{2}-S_{2} w_{1}}{\left(w_{1}+S_{1}\right)+\left(w_{2}+S_{2}\right)} .
$$




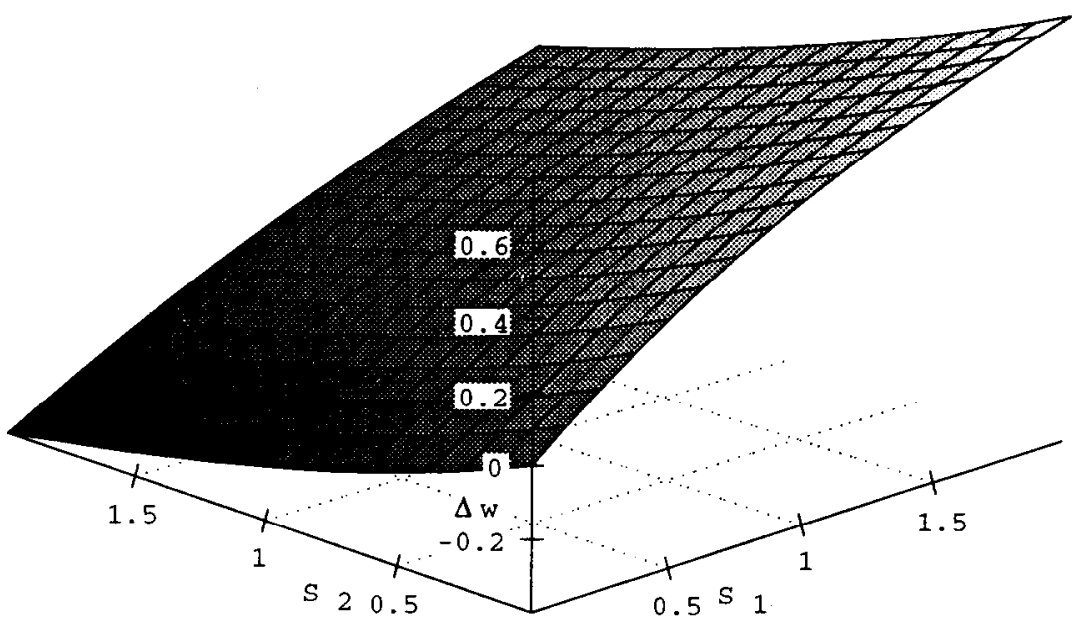

FIG. 2. A plot of Equation (4) with $w_{1}=w_{2}=1$ for various values of $S_{1}$ and $S_{2}$. The plot represents a hyperbolic plane of $\Delta w$.

If $\Delta w$ is plotted against the additional weights $S_{1}$ and $S_{2}$, then the resulting plot shows a hyperbolic plane for $\Delta w$ (see Figure 2).

The calculated values for $\Delta w$ from Equation (4) were compared to the experimental values of the ratio of the jnd and to the magnitudes of the total stimuli from Weber [1]. As shown in Table 1, the calculated values of $\Delta w$ are identical to the experimental values of Weber [1]. When $w$ is $1.03, \Delta w$ becomes equal in magnitude to the jnd $/\left(S_{1}+S_{2}\right)$ values. It is interesting that such a simple physical system demonstrates Weber's law, which describes a wide variety of complex sensory systems.

\section{TABLE 1}

Comparison of Weber's Experimental Observation to the Values of $\Delta w$ Calculated by Equation (4)

\begin{tabular}{cccc}
\hline $\begin{array}{c}\text { Weber's exp. } \\
S_{2} / S_{1}(\mathrm{~g})\end{array}$ & $\begin{array}{c}\text { jnd }^{\mathrm{a}} \\
(\mathrm{g})\end{array}$ & jnd $/\left(S_{1}+S_{2}\right)$ & $\Delta w^{\mathrm{b}}$ \\
\hline $20 / 21$ & 1 & 0.024 & 0.024 \\
$40 / 42$ & 2 & 0.024 & 0.024 \\
$60 / 63$ & 3 & 0.024 & 0.024 \\
$80 / 84$ & 4 & 0.024 & 0.024 \\
$100 / 105$ & 5 & 0.024 & 0.024 \\
\hline
\end{tabular}

a jnd, just noticeable difference.

${ }^{b}$ For $w_{1}=w_{2}=1.03$. 


\section{DISCUSSION}

Weber's law is strictly obeyed by a physical beam balance as shown by the data in Table 1 . The mathematical representation of the beam balance model to Weber's law provides for a more intuitive understanding of the relationship of Weber's law to sensory and receptor systems. This model may also offer alternative methods to compute the perturbations that result from unequal effects on coupled steady-state systems. Interestingly, the beam balance model displays a loss of response at larger loads and can be used as a physical model of receptor desensitization [4]. A practical outgrowth from this work is that a relatively simple mathematical description models sensory phenomena and may aid in the understanding of sensory and receptor systems.

Stevens examined a number of sensory systems and concluded that great importance must be placed upon the transducer process itself, at the periphery [5]. In particular, Stevens wondered how it could be that the receptor process bends the sensory function by a ratio-preserving compression and thereby permits the coupling of the organism to such dynamic ranges of stimuli. Current research based upon the beam balance model [4] may provide the mathematical framework for further insights into this important question.

\section{REFERENCES}

1 E. Batschelet, Introduction to Mathematics for Life Scientists, Springer-Verlag, New York, 1979, pp. 157-162.

2 S. M. Dawis, A molecular basis for Weber's law, Visual Neurosci. 7:285-320 (1991).

3 H. Hatze, A teleological explanation of Weber's law and the motor unit size law, Bull. Math. Biol. 41:407-425 (1979).

4 R. G. Lanzara, Drug composition to prevent desensitization of cellular receptors, Patent pending, 1992.

5 S. S. Stevens, Neural events and the psychophysical law, Science 170:1043-1050 (1970). 